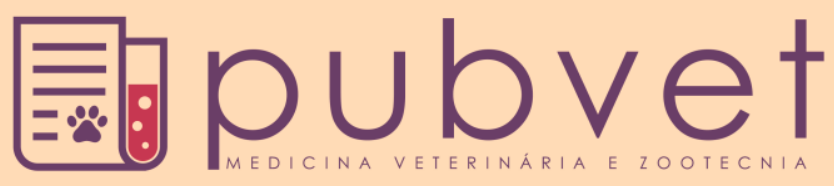

https://doi.org/10.31533/pubvet.v12n9a177.1-6

\title{
Taxa de prenhez de novilhas de corte submetidas à IATF com protocolo a base de benzoato de estradiol ou Ovsynch modificado
}

\author{
Carlos Santos Gottschall ${ }^{1 *} \bullet$, Leonardo Rocha da Silva ${ }^{2} \bullet$ \\ ${ }^{I}$ Professor dos cursos de Medicina Veterinária e Agronomia da Universidade Luterana do Brasil. Canoas, RS, Brasil. E-mail: carlosgott@ cpovo.net \\ ${ }^{2}$ Mestrando em Ciências na área de Produção Animal, Universidade Federal de Pelotas, Pelotas, RS, Brasil. E-mail: Leonardor.s@outlook.com \\ *Autor para correspondência.
}

\begin{abstract}
RESUMO. O presente estudo avaliou o desempenho reprodutivo de 217 novilhas de corte submetidas a diferentes protocolos de inseminação artificial em tempo fixo (IATF), seguidos pelo repasse com touros. Os animais foram distribuídos aleatoriamente em três grupos, de acordo com o protocolo ao qual foram expostos. $O$ grupo 1 (G1; 118 novilhas) foi submetido a um protocolo modificado de Ovsynch, com inclusão de um dispositivo de progesterona $\left(\mathrm{P}_{4}\right) . \mathrm{O}$ grupo 2 (G2; 50 novilhas) diferiu apenas no hormônio indutor da ovulação, sendo utilizado o benzoato de estradiol (BE). O grupo 3 (G3; 49 novilhas) foi submetido a um protocolo com $\mathrm{BE}, \mathrm{P}_{4}$ e prostaglandina $\left(\mathrm{PGF}_{2} \alpha\right)$. Sete dias após a IATF, todas as novilhas foram expostas a touros para repasse. As análises estatísticas foram realizadas pelos métodos Qui-quadrado e de variância $(\mathrm{P}=0,05)$. A taxa de prenhez à IATF do $\mathrm{G} 1$ foi superior $(57,6 \% ; \mathrm{P}<0,05)$ aos grupos G2 $(34,0 \%)$ e G3 $(34,7 \%)$ que não diferiram entre si. A prenhez final não diferiu entre os grupos. O protocolo do G1, modificado de Ovsynch pela inclusão de um dispositivo de $\mathrm{P}_{4}$ demonstrou melhor desempenho para novilhas de corte. A taxa de prenhez final não sofreu interferência da prenhez à IATF.
\end{abstract}

Palavras Chave: Estradiol, GnRH, Ovsynch, Progesterona, Taxa de prenhez

\section{Reproductive performance in beef heifer submitted to FTAI with protocol BE-base or Ovsynch modified}

\begin{abstract}
The reproductive performance of 217 beef heifers submitted to different FTAI protocols and clean up bulls was evaluated. The animals were randomly divided in three groups. The group 1 (G1; 118 heifers) was exposed to a protocol modified of $O v s y n c h$, with inclusion of a progesterone device $\left(\mathrm{P}_{4}\right)$. The group $2(\mathrm{G} 2 ; 50$ heifers) was differed the $\mathrm{G} 1$ to application of estradiol benzoate (EB) as ovulation inducer. The group 3 (G3; 49 heifers) was submitted to a protocol with $\mathrm{EB}, \mathrm{P}_{4}$ and $\mathrm{PGF}_{2} \alpha$. Seven days after FTAI, all heifers were exposed to bulls. The statistical analyzes were performed using Chi-square and variance methods $(\mathrm{P}=0.05)$. The pregnancy rate to FTAI was higher by G1 $(57,6 \%$; P < 0.05) than G2 (34.0\%) and G3 (34.7\%), which did not differ. The final pregnancy rate did not differ among groups. The G1 group protocol was superior than $\mathrm{G} 2$ and $\mathrm{G} 3$ when evaluating the pregnancy rate. The final pregnancy rate did not differ among groups, regardless of the protocol used.
\end{abstract}

Keywords: Estradiol, GnRH, Ovsynch, progesterone, pregnancy rate

\section{Tasa de preñez de novillas de carne sometidas a IATF con protocolo a base de benzoato de estradiol o Ovsynch modificado}

RESUMEN. El presente estudio evaluó el desempeño reproductivo de 217 novillas de carne sometidas a diferentes protocolos de inseminación artificial en tiempo fijo (IATF), seguidos por 
el repase con toros. Los animales fueron distribuidos aleatoriamente en tres grupos, de acuerdo con el protocolo al cual fueron expuestos. El grupo 1 (G1, 118 novillas) fue sometido a un protocolo modificado de Ovsynch, con un dispositivo de progesterona $\left(\mathrm{P}_{4}\right)$. El grupo $2(\mathrm{G} 2,50$ novillas) difirió sólo en la hormona inductora de la ovulación, siendo utilizado el benzoato de estradiol (BE). El grupo 3 (G3, 49 novillas) fue sometido a un protocolo con $\mathrm{BE}, \mathrm{P}_{4} \mathrm{y}$ prostaglandina $\left(\mathrm{PGF}_{2} \alpha\right)$. Siete días después de la IATF, todas las novillas fueron expuestas a toros para el repase. Los análisis estadísticos fueron realizados por los métodos Qui-cuadrado y de varianza $(\mathrm{P}=0,05)$. La tasa de preñez a la IATF del $\mathrm{G} 1$ fue superior $(57,6 \%, \mathrm{P}<0,05)$ a los grupos G2 $(34,0 \%)$ y G3 $(34,7 \%)$, que no diferían entre sí. La preñez final no difirió entre los grupos. El protocolo del G1, modificado de Ovsynch por la inclusión de un dispositivo de $\mathrm{P}_{4}$, demostró un mejor rendimiento para las novillas de carne. La tasa de preñez final no sufrió interferencia de la preñez a la IATF.

Palabras clave: Estradiol, GnRH, Ovsynch, progesterona, tasa de preñez

\section{Introdução}

O ciclo estral de bovinos pode ser controlado com o emprego de diversos protocolos, utilizando diferentes fármacos e formas de aplicação (Baruselli et al., 2012; Gottschall et al., 2009). No entanto, as diferentes associações farmacológicas disponíveis para a realização da inseminação artificial em tempo fixo (IATF) podem resultar em respostas variadas (Ferreira et al., 2013). Os tratamentos que têm demonstrado melhores resultados são os que congregam um dispositivo de progesterona $\left(\mathrm{P}_{4}\right)$, aplicação de análogos de estradiol $\left(\mathrm{E}_{2}\right)$ e prostaglandina $\left(\mathrm{PGF}_{2} \alpha\right)$ (Baruselli et al., 2004; Bó et al., 2002; Cutaia, 2006) ou, ainda, o protocolo conhecido como Ovsynch (Pursley et al., 1995). Este segundo, menos usado no Brasil, baseia-se na aplicação de duas doses do hormônio liberador de gonadotrofinas $(\mathrm{GnRH})$ intercaladas por uma de $\mathrm{PGF}_{2} \alpha$.

A eficiência reprodutiva dos protocolos que utilizam GnRH são dependentes da presença de um folículo dominante ao início do tratamento (Santos \& Vasconcelos, 2008). A ovulação estimulada pelo GnRH cursa com a produção e secreção de $\mathrm{P}_{4}$ endógena em concentrações suficientes para controlar o crescimento folicular subsequente. Patterson et al. (2003) mencionam que, apenas a luteinização de folículos menos desenvolvidos não é capaz de realizar este controle. Desta forma, os protocolos que associam $\mathrm{E}_{2}$ e dispositivos de $\mathrm{P}_{4}$ sofrem menor interferência da fase do ciclo estral dos animais ao início do tratamento (Baruselli et al., 2004; Bó et al., 2003).

Como alternativa aos animais que não apresentem um folículo dominante ao início de protocolos com GnRH, Martínez et al. (2002) sugerem o uso de um dispositivo de $\mathrm{P}_{4}$, a fim de complementar o aporte endógeno de $\mathrm{P}_{4}$.
Entretanto, Silva \& Gottschall (2014) mencionam que estes dispositivos devem conter baixa concentração de $\mathrm{P}_{4}$ para não prejudicar animais que apresentem um folículo dominante ao início do tratamento.

O objetivo do presente trabalho foi avaliar a resposta reprodutiva de novilhas de corte após a aplicação de três diferentes protocolos para IATF e repasse com touros.

\section{Material e Métodos}

O presente experimento foi previamente avaliado e aprovado pela Comissão de Ética no Uso de Animais da ULBRA (CEUA - ULBRA), registrado com o número de protocolo: 2013-25P.

Foram avaliados os dados reprodutivos de 217 novilhas de corte, da raça Braford e cruzas, entre 24 e 26 meses de idade submetidas à IATF e repasse com touros. Todas as novilhas foram pesadas individualmente e tiveram a condição corporal (CC) estimada em uma escala de 1 (muito magra) a 5 (muito gorda) (Tabela 1). No primeiro grupo (G1 - 118 novilhas) foi aplicado o protocolo Ovsynch, modificado pela inclusão de um dispositivo intravaginal de $\mathrm{P}_{4}$ (DIP) reutilizado por duas vezes ( $3^{\circ}$ uso), com 1 grama de concentração quando novo (Figura 1). O protocolo aplicado no segundo grupo (G2 - 50 novilhas) diferiu ao grupo G1 apenas pelo hormônio indutor da ovulação e momento da IATF. Para indução e sincronização da ovulação se substituiu a segunda aplicação do $\mathrm{GnRH}$ por uma de benzoato de estradiol (BE) (Figura 2). O terceiro grupo (G3 49 novilhas) foi submetido a um protocolo com $\mathrm{BE}, \mathrm{P}_{4}$ e $\mathrm{PGF}_{2} \alpha$. Neste grupo, o DIP utilizado foi de uso único, com concentração de 0,75 gramas (Figura 3). O sêmen utilizado para a IATF foi proveniente de três diferentes touros, distribuídos 
ao acaso entre os tratamentos, sem diferença estatística entre eles $(\mathrm{P}>0,05)$.

Tabela 1. Dados de peso, CC e número de animais por grupo.

\begin{tabular}{cccc}
\hline Grupo & $\mathrm{N}$ & Peso $(\mathrm{kg})$ & $\mathrm{CC}(1-5)$ \\
\hline G1 & 118 & 315 & 3,2 \\
G2 & 50 & 319 & 3,2 \\
G3 & 49 & 326 & 3,2 \\
\hline
\end{tabular}

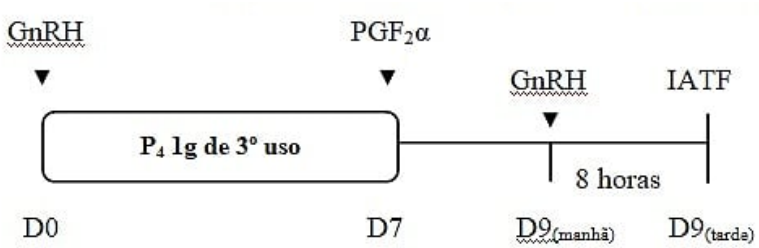

Figura 1. Fluxograma do protocolo aplicado no grupo G1.

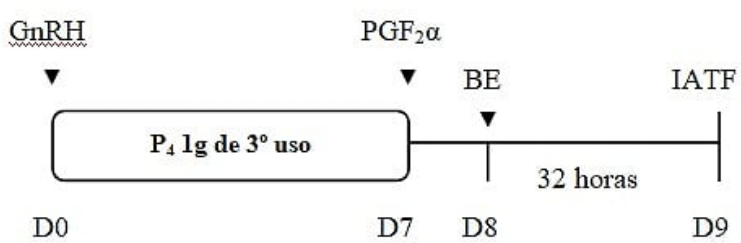

Figura 2. Fluxograma do protocolo aplicado no grupo G2.

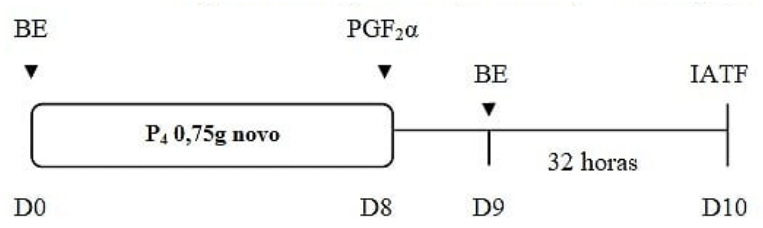

Figura 3. Fluxograma do protocolo aplicado no grupo G3.

\section{Resultados e discussão}

As novilhas do grupo G1 apresentaram taxa de prenhez à IATF superior aos demais grupos $(\mathrm{P}<$ $0,05)$, ao passo que, os grupos G2 e G3 não diferiram entre si (Figura 4). O número de novilhas prenhas de inseminação foi $68(118)$, 17(50) e 17(49), respectivamente para os grupos G1, G2 e G3. Os grupos não apresentaram diferença para a taxa de prenhez final (Figura 4), com número de novilhas prenhes ao final da estação de acasalamento de 109 (118) para G1, 43 (50) para G2 e 44 (49) para G3.

O protocolo aplicado nos animais do grupo G3 $\left(\mathrm{BE}+\mathrm{P}_{4}+\mathrm{PGF}_{2} \alpha\right)$, assim como seus similares, tem sido amplamente difundido no Brasil. Diversos estudos demonstram melhor desempenho reprodutivo destes em comparação aos protocolos que utilizam GnRH (Baruselli et al., 2004; $\underline{\text { Bó et }}$ al., 2003; Bó et al., 2002). Em uma revisão realizada por Bó et al. (2003) os autores ressaltam que há uma grande variabilidade no desempenho de protocolos com $\mathrm{GnRH}$, principalmente para novilhas de corte. Pursley et al. (1995), precursores do protocolo Ovsynch observaram considerável disparidade de resultados entre vacas e novilhas leiteiras.

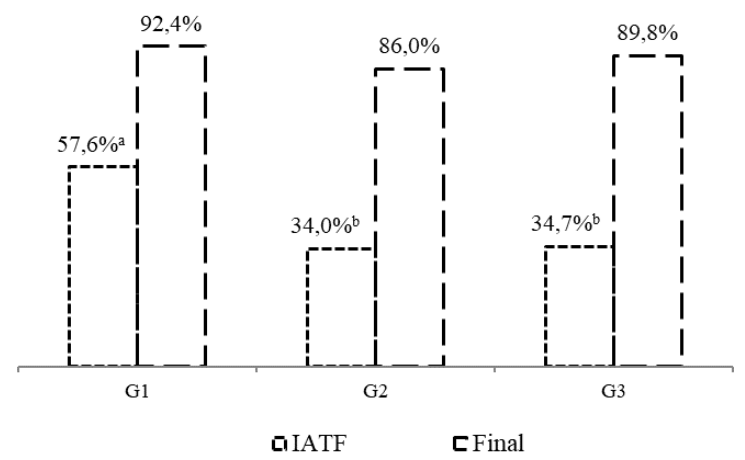

Figura 4. Taxa de prenhez à IATF e final por grupo de protocolo

A ausência de um folículo dominante cursa com a manifestação estral de animais antes do término dos protocolos com $\mathrm{GnRH}$, principal limitante destes tratamentos. Patterson et al. (2003) observaram que apenas $15 \%$ das vacas apresentaram estro entre a primeira aplicação do GnRH e a aplicação de $\mathrm{PGF}_{2} \alpha$. Pursley et al. (1995) observaram alta incidência de ovulação após a primeira aplicação do GnRH em vacas de leite $(90 \%)$. No entanto, nas novilhas, a taxa de ovulação chegou a $56 \%$. Quando as concentrações de $\mathrm{P}_{4}$ atingem níveis sub-luteais há o aumento da frequência pulsátil de $\mathrm{LH}$, estimulo à maturação folicular e presença do estro (Baruselli et al., 2004).

Alguns autores vêm avaliando diferentes modificações para tornar os protocolos com GnRH menos dependentes da fase do ciclo estral (Gottschall \& Silva, 2012; Martínez et al., 2002; Silva \& Gottschall, 2014). Martínez et al. (2002) avaliaram a taxa de prenhez de novilhas de corte submetidas aos protocolos Ovsynch, Co-synch e suas modificações. Em um dos experimentos, novilhas foram submetidas ao protocolo Co-synch tradicional e modificado pela inclusão de um DIP (CIDR-B). O protocolo Co-synch tradicional resultou em taxa de prenhez de $39 \%$, enquanto que no protocolo modificado esta foi de $68 \%$ (P < $0,05)$. Em outro experimento, também em novilhas de corte, os mesmos autores avaliaram o desempenho do protocolo Ovsynch tradicional, modificado com DIP e um terceiro similar ao do 
grupo G3 $\left(\mathrm{E}_{2}+\mathrm{P}_{4}+\mathrm{PGF}_{2} \alpha\right)$. No entanto, neste último, uma dose de $100 \mathrm{mg} / \mathrm{im}$ de $\mathrm{P}_{4}$ foi aplicada no momento da inserção do DIP e nos dois primeiros o $\mathrm{GnRH}$ foi substituído por aplicações do hormônio luteinizante da porca $(\mathrm{pLH})$. A taxa de prenhez para os respectivos grupos foi de $37,5 \%, \quad 64,7 \%$ e $75,0 \%$, com significativa diferença $(\mathrm{P}<0,04)$ entre o primeiro (Ovsynch tradicional) e o último $\left(\mathrm{E}_{2}+\mathrm{P}_{4}+\mathrm{PGF}_{2} \alpha\right)$ protocolo, que não diferiram do segundo $\left(\right.$ Ovsynch $\left.+\mathrm{P}_{4}\right)$.

Neste experimento citado, o protocolo do último grupo, a base de $\mathrm{BE}$, resultou em taxa de prenhez superior a do grupo G3 do presente trabalho, com protocolo similar. Entretanto, se deve levar em consideração a concentração de $\mathrm{P}_{4}$ que as novilhas foram expostas. Estudos sugerem que altas concentrações de $\mathrm{P}_{4}$ favorecem novilhas acíclicas (Rasby et al., 1998). De forma contrária, as novilhas do grupo Ovsynch modificado podem ter sido prejudicadas pelo excesso de $\mathrm{P}_{4}$, já que associou um DIP novo e de elevada concentração (CIDR-B) ao uso de GnRH. O uso do GnRH deve antever a reutilização dos dispositivos (Gottschall \& Silva, 2012; Silva \& Gottschall, 2014). A aplicação do GnRH culmina com a produção, mesmo que baixa, de $\mathrm{P}_{4}$ endógena proveniente de folículos luteinizados (Patterson et al., 2003). Desta forma, o dispositivo reutilizado suplementa a $\mathrm{P}_{4}$ dos animais que luteinizam folículos menores e não prejudica animais que ovulam.

A consistência e o volume de dados oriundos de experimentos com associação entre $\mathrm{GnRH}$ e $\mathrm{P}_{4}$ vêm sendo evidenciada há alguns anos (Gottschall \& Silva, 2012; Mapletoft et al., 2003; Martínez et al., 2002; Martínez et al., 2002; Martínez et al., 2004; Patterson et al., 2003; Silva \& Gottschall, 2014). Gottschall \& Silva (2012) avaliaram a taxa de prenhez de 196 novilhas submetidas à IATF após IA convencional. As novilhas foram expostas a dois protocolos de IATF idênticos aos dos grupos $\mathrm{G} 1\left(\right.$ Ovsynch $\left.+\mathrm{P}_{4}\right)$ e $\mathrm{G} 3\left(\mathrm{BE}+\mathrm{P}_{4}+\mathrm{PGF}_{2} \alpha\right)$ do presente trabalho. Os autores observaram taxa de prenhez de $46,7 \%$ para o protocolo Ovsynch modificado e $30,1 \%$ no protocolo a base de BE (P $<0,05)$. Houve diferença de aproximadamente 11 pontos percentuais na taxa de prenhez entre os protocolos Ovsynch $+\mathrm{P}_{4}$ destes autores e o do grupo $\mathrm{G} 1$ do presente trabalho $(46,7 \%$ e $57,6 \%$, respectivamente). Entretanto, os animais experimentados no trabalho citado acima representavam, com exceção das possíveis falhas de observação de cio, animais acíclicos.
Em outro experimento, Silva \& Gottschall (2014) avaliaram 368 novilhas submetidas à IATF com protocolo similar ao do grupo G3 do presente trabalho $\left(\mathrm{BE}+\mathrm{P}_{4}+\mathrm{PGF}_{2} \alpha\right)$, denominado PEPE1, um segundo que diferiu no tipo e uso do DIP, denominado PEPE2, e um terceiro protocolo idêntico ao do grupo $\mathrm{G} 1\left(\right.$ Ovsynch $\left.+\mathrm{P}_{4}\right)$ do presente trabalho, denominado OVSYNCH+P4. Neste trabalho, os resultados apresentaram-se mais homogêneos entre os grupos. A taxa de prenhez à IATF para os respectivos grupos foi de $46,5 \%$, $43,1 \%$ e $48,0 \%$, sem diferença estatística.

Ao analisar todos os experimentos supracitados, a taxa de prenhez dos protocolos com $\mathrm{E}_{2}$ e $\mathrm{P}_{4}$ variou de 30,1\% (Gottschall \& Silva, 2012) a 75\% (Martínez et al., 2002), com uma amplitude de 45 pontos percentuais. Já os tratamentos que associaram um dispositivo de $\mathrm{P}_{4}$ aos protocolos com GnRH apresentaram variabilidade na taxa de prenhez de apenas 21 pontos percentuais, de $46,7 \%$ (Gottschall \& Silva, 2012) a 68,0\% (Martínez et al., 2002). A amplitude na variação da taxa de prenhez do protocolo Ovsynch $+\mathrm{P}_{4}$ representa menos da metade da observada nos protocolos à base de $\mathrm{E}_{2}$ e $\mathrm{P}_{4}$. Os dados apresentados, associados aos resultados obtidos no presente trabalho, vão de encontro com o observado por Bó et al. (2003) em que constatam que protocolos GnRH-base são inconsistentes nos resultados de prenhez. Entretanto, deve-se considerar a modificação do protocolo pela inclusão do DIP.

A redução nos custos de produção, sem interferência no desempenho produtivo, significa melhora na eficiência econômico do sistema. Desta forma, alguns estudos objetivaram avaliar o desempenho reprodutivo de protocolos modificados, que agreguem melhor custo/benefício à técnica (Câmara et al., 2009; Dickson et al., 2012; Lima et al., 2010; Mapletoft et al., 2003; Martínez et al., 2004; Yániz et al., 2004). O presente experimento avaliou a substituição da segunda aplicação do GnRH por uma de BE em protocolo modificado de Ovsynch. $\mathrm{O}$ BE mostrou-se menos eficaz que o GnRH como indutor da ovulação, quando avaliada a resposta pela taxa de prenhez. Lima et al. (2010) avaliaram uma modificação similar, no entanto, em um protocolo Ovysynch tradicional (Heatsynch) em vacas leiteiras. Estes autores observaram redução na taxa de prenhez com protocolo Heatsynch, sugerindo que o $\mathrm{GnRH}$ teria sincronizado a ovulação de forma mais efetiva que $\mathrm{o} B E$. Contudo, Dickson et al. (2012) com a mesma 
modificação, não observaram efeito significativo sobre a taxa de prenhez. Quando avaliando o desempenho reprodutivo de novilhas de corte submetidas a diferentes hormônios indutores de ovulação $(\mathrm{GnRH} x \mathrm{BE})$ em um protocolo à base de BE, Martinez et al. (2004) observaram significativa diferença $(P<0,02)$ na taxa de prenhez, com superioridade para o protocolo que utilizou GnRH (59,8\% x 50,0\%). Este trabalho vai ao encontro do presente estudo, podendo sugerir que, em novilhas de corte, o GnRH tem melhor capacidade para induzir e sincronizar a ovulação que o BE.

O repasse de touros após a IATF complementa a taxa de prenhez de novilhas de corte, com equivalência estatística, independente do protocolo utilizado (Gottschall \& Silva, 2012; Silva \& Gottschall, 2014). No entanto, apesar de não haver diferença estatística, Gottschall \& Silva (2012) observaram taxa de prenhez final de 80,0\% para o protocolo modificado de Ovsynch $\left(\mathrm{GnRH}+\mathrm{P}_{4}\right)$ e $68,4 \%$ para o protocolo PEPE $\left(\mathrm{BE}+\mathrm{P}_{4}+\mathrm{PGF}_{2} \alpha\right)$, com considerável diferença numérica. É possível observar nestes dados que, embora a taxa de prenhez final mais que dobre em relação a taxa de prenhez à IATF do protocolo PEPE $(30,1 \%$ para $68,4 \%)$, a diferença na taxa de prenhez final foi de aproximadamente 12 pontos percentuais entre os protocolos, número que na produção de bovinos de corte não pode ser desprezado. Estes dados reforçam a importância de obter bons índices de prenhez já nos primeiros dias da estação de acasalamento, oportunizando, desta forma, a obtenção de um número maior de concepções.

\section{Conclusões}

A modificação do protocolo Ovsynch, associando-o a um DIP com baixas concentrações de $\mathrm{P}_{4}$, resulta em taxa de prenhez à IATF superiores para novilhas de corte em comparação a protocolos com BE. Este protocolo pode ser uma alternativa adicional ao terceiro uso de DIP de 1 grama, quando novos.

A modificação do hormônio indutor da ovulação no protocolo Ovsynch, mesmo associado à $\mathrm{P}_{4}$, não demonstrou ser uma opção viável quando feita com BE. Entretanto, sugere-se que esta modificação seja mais bem estudada.

O repasse com touros compensa estatisticamente a diferença da prenhez à IATF, independente do protocolo utilizado.

\section{Referências Bibliográficas}

Baruselli, P. S., Reis, E. L., Marques, M. O., Nasser, L. F., \& Bó, G. A. 2004. The use of hormonal treatments to improve reproductive performance of anestrous beef cattle in tropical climates. Animal Reproduction Science, 82, 479-486.

Baruselli, P. S., Sales, J. N. S., Sala, R. V., Vieira, L. M., \& Sá Filho, M. F. 2012. History, evolution and perspectives of timed artificial insemination programs in Brazil. Animal Reproduction, 9(3), 139-152.

Bó, G. A., Baruselli, P. S., \& Martınez, M. F. 2003. Pattern and manipulation of follicular development in Bos indicus cattle. Animal Reproduction Science, 78(3-4), 307-326.

Bó, G. A., Cutaia, L., \& Tribulo, R. 2002. Tratamientos hormonales para inseminación artificial a tiempo fijo en bovinos para carne: algunas experiencias realizadas en Argentina. Primera Parte. Sitio Argentina de Producción Animal, 4, 1-14.

Câmara, D. R., Figueira, R. F., Mendonça, L. B. R., \& Morais, C. S. M. 2009. Redução hormonal em protocolo Ovsynch para inseminação artificial em tempo fixo em fêmeas Nelore. Revista Brasileira de Ciências Agrárias, 4(4), 467-471.

Cutaia, L. E. 2006. Programas de inseminación artificial a tiempo fijo: análisis de costos e implementación. Sitio Argentina de Producción Animal 1, 1-15.

Dickson, N., Siew, N., Singh-Knights, D., Bourne, G., \& Knights, M. 2012. Technical and economic outcome using various timed artificial insemination protocols in dairy cattle in the tropics. Journal of Animal Production Advances, 2(1), 490-499.

Ferreira, M. C. N., Miranda, R., Figueiredo, M. A., Costa, O. M., \& Palhano, H. B. 2013. Impacto da condição corporal sobre a taxa de prenhez de vacas da raça nelore sob regime de pasto em programa de inseminação artificial em tempo fixo (IATF). Semina: Ciências Agrárias, 34(4), 1861-1868.

Gottschall, C. S., Bittencourt, H. R., Mattos, R. C., \& Gregory, R. M. 2009. Antecipação da aplicação de prostaglandina, em programa de inseminação artificial em tempo fixo em vacas de corte. Revista Brasileira de Saúde e Produção Animal, 10(4), 970-979. 
Gottschall, C. S., \& Silva, L. R. 2012. Resposta reprodutiva de novilhas de corte aos dois e três anos de idade submetidas a diferentes protocolos para inseminação artificial em tempo fixo (IATF). Veterinária em Foco, 10(1), 16-25.

Lima, F. A., Veras, M. B., Sales, J. N. S., Crepaldi, G. A., Maio, J. R. G., \& Baruselli, P. S. 2010. Redução da primeira dose de GnRH em vacas holandesas de alta produção sincronizadas com Ovsynch ou Heatsynch. Brazilian Journal of Veterinary Research and Animal Science, 47(2), 125-131.

Mapletoft, R. J., Martinez, M. F., Colazo, M. G., \& Kastelic, J. P. 2003. The use of controlled internal drug release devices for the regulation of bovine reproduction. Journal of Animal Science, 81(14-Sup. 2), E28-E36.

Martinez, F., Kaabi, M., Martinez-Pastor, F., Alvarez, M., Anel, E., Boixo, J. C., . . . Anel, L. 2004. Effect of the interval between estrus onset and artificial insemination on sex ratio and fertility in cattle: a field study. Theriogenology, 62(7), 1264-1270.

Martínez, M. F., Kastelic, J. P., Adams, G. P., Cook, B., Olson, W. O., \& Mapletoft, R. J. 2002. The use of progestins in regimens for fixed-time artificial insemination in beef cattle. Theriogenology, 57(3), 1049-1059.

Martínez, M. F., Kastelic, J. P., Adams, G. P., \& Mapletoft, R. J. 2002. The use of a progesterone-releasing device (CIDR-B) or melengestrol acetate with $\mathrm{GnRH}$, $\mathrm{LH}$, or estradiol benzoate for fixed-time AI in beef heifers. Journal of Animal Science, 80(7), 1746-1751.

Martínez, M. F., Kastelic, J. P., \& Mapletoft, R. J. 2004. The use of estradiol and/or GnRH in a two-dose PGF protocol for breeding management of beef heifers. Theriogenology, 62(1-2), 363-372.

Patterson, D. J., Kojima, F. N., \& Smith, M. F. 2003. A review of methods to synchronize estrus in replacement beef heifers and postpartum cows. Journal of Animal Science, 81(14), E166-E177.

Pursley, J. R., Mee, M. O., \& Wiltbank, M. C. 1995. Synchronization of ovulation in dairy cows using PGF $2 \alpha$ and GnRH. Theriogenology, 44(7), 915-923.

Rasby, R. J., Day, M. L., Johnson, S. K., Kinder, J. E., Lynch, J. M., Short, R. E., . . Hafs, H. D. 1998. Luteal function and estrus in peripubertal beef heifers treated with an intravaginal progesterone releasing device with or without a subsequent injection of estradiol. Theriogenology, 50(1), 55-63.

Santos, R. M., \& Vasconcelos, J. L. M. 2008. Eficácia da dose reduzida de gonadorelina e diferentes prostaglandinas no protocolo ovsynch em vacas holandesas. Arquivo Brasileiro de Medicina Veterinária e Zootecnia, 60(6), 1323-1328.

Silva, L. R., \& Gottschall, C. S. 2014. Desempenho reprodutivo de novilhas de corte submetidas a diferentes protocolos para inseminação artificial em tempo fixo (IATF). Revista de Iniciação Científica da ULBRA, l(12), 5-13.

Yániz, J. L., Murugavel, K., \& López-Gatius, F. 2004. Recent developments in oestrous synchronization of postpartum dairy cows with and without ovarian disorders. Reproduction in Domestic Animals, 39(2), 86-93.

Recebido: 12 Julho, 2018.

Aprovado: 22 Agosto, 2018

Publicado: 10 Setembro, 2018

Licenciamento: Este artigo é publicado na modalidade Acesso Aberto sob a licença Creative Commons Atribuição 4.0 (CC-BY 4.0), a qual permite uso irrestrito, distribuição, reprodução em qualquer meio, desde que o autor e a fonte sejam devidamente creditados. 